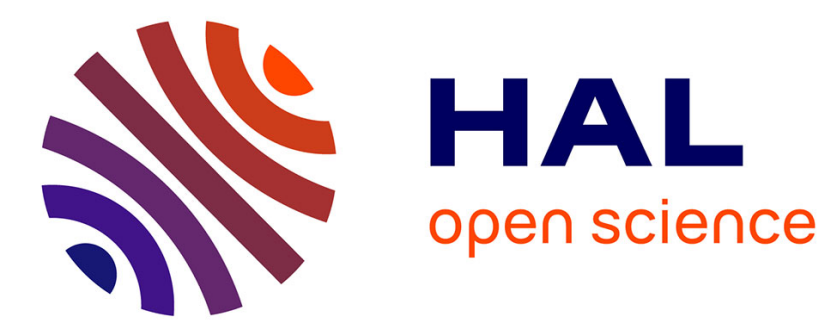

\title{
Vieillissement de l'organisation conceptuelle : accès aux propriétés des objets naturels et fabriqués
}

Nadège Roll-Carpentier, Françoise Bonthoux, Solène Kalénine

\section{To cite this version:}

Nadège Roll-Carpentier, Françoise Bonthoux, Solène Kalénine. Vieillissement de l'organisation conceptuelle: accès aux propriétés des objets naturels et fabriqués. Annee Psychologique, 2006, 106 (3), pp.27-47. 10.4074/s0003503306002028 . hal-00188550

\section{HAL Id: hal-00188550 \\ https://hal.science/hal-00188550}

Submitted on 3 Dec 2007

HAL is a multi-disciplinary open access archive for the deposit and dissemination of scientific research documents, whether they are published or not. The documents may come from teaching and research institutions in France or abroad, or from public or private research centers.
L'archive ouverte pluridisciplinaire $\mathbf{H A L}$, est destinée au dépôt et à la diffusion de documents scientifiques de niveau recherche, publiés ou non, émanant des établissements d'enseignement et de recherche français ou étrangers, des laboratoires publics ou privés. 
Vieillissement de l'organisation conceptuelle : accès aux propriétés

des objets naturels et fabriqués

Nadège Roll-Carpentier ${ }^{1}$, Françoise Bonthoux et Solène Kalénine

Laboratoire de Psychologie et NeuroCognition

CNRS UMR 5105, Université Pierre Mendes France, Grenoble, France

\footnotetext{
${ }^{1}$ Correspondance : Nadège Roll-Carpentier, Laboratoire de Psychologie et NeuroCognition. Université PierreMendes France. PO Box 47 - 38040 GRENOBLE CEDEX 9 (France) Tél. 33 (0)4 76825630 / Fax 33 (0)4 76 8278 34. nadege.roll-carpentier@upmf-grenoble.fr
} 


\section{RESUME}

Les connaissances conceptuelles seraient organisées chez l'adulte par le domaine d'appartenance des objets (naturels ou fabriqués), le type de propriétés (visuelles ou fonctionnelles) et leur niveau de spécificité. Cette recherche vise à évaluer les propriétés les plus sensibles au vieillissement. Leur accessibilité est testée chez des adultes jeunes et âgés en fonction de ces variables, à l'aide d'une épreuve de vérification de propriétés. Dans les deux groupes, les propriétés fonctionnelles sont plus accessibles que les propriétés visuelles, surtout à un niveau spécifique et pour les objets fabriqués. Une difficulté particulière pour les propriétés visuelles spécifiques des objets naturels se manifeste lors du vieillissement, ce qui suggère une forme commune d'altération entre le vieillissement normal et la démence Alzheimer. Les résultats liés au vieillissement sont discutés vis-à-vis des hypothèses de formation des concepts d'objets.

Mots clés : concepts, vieillissement, propriétés, objets naturels, objets fabriqués

SUMMARY: Conceptual organization and aging: properties accessibility for natural objects and artifacts

The organization of adults' conceptual knowledge relies on three main factors: semantic domain (natural objects or artifacts), type of property (visual or functional), and specificity level (general or distinctive properties). This study aims at evaluating which object properties are the most sensitive to normal aging. Accessibility of properties was assessed in young and old adults as a function of these variables, using a property verification task. In both groups, functional properties were more quickly accessed than visual ones, especially specific properties of artifacts. A particular difficulty for specific visual properties of natural objects appeared with aging, suggesting a common form of decline between normal aging and dementia of Alzheimer type. Results related to aging are discussed in relation to hypotheses of the formation of objects concepts.

Key words: concepts, aging, properties, living, non living 
Vieillissement de l'organisation conceptuelle : accès aux propriétés des objets naturels et fabriqués

\section{Introduction}

Certains patients, atteints notamment d'encéphalite herpétique, montrent un déficit pour les objets naturels, associé à une relative préservation des objets fabriqués (Warrington et Shallice, 1984) ou, plus rarement, le profil inverse (Warrington et McCarthy, 1983, 1987). Les catégories impliquées dans cette double dissociation comprennent le plus souvent les animaux, les végétaux et la nourriture pour les objets naturels et les outils, les véhicules, les meubles et les ustensiles de cuisine pour les objets fabriqués. Une hypothèse maintenant classique, la «théorie sensorielle - fonctionnelle » (SFT) avancée par Warrington et Shallice (1984), est que ces déficits seraient associés à une plus ou moins grande pondération des propriétés sensorielles ou fonctionnelles. Pour ces auteurs, les objets naturels seraient plus fortement caractérisés par leurs propriétés perceptives/visuelles que par leurs propriétés fonctionnelles/associatives alors qu'à l'inverse, les objets fabriqués seraient surtout caractérisés par leurs propriétés fonctionnelles. Le système sémantique pourrait alors se fractionner en deux réseaux, l'un reliant les concepts surtout définis par leur aspect et l'autre, les concepts essentiellement définis par leur usage. A l'heure actuelle, les résultats des patients cérébro-lésés ne semblent pas corroborer directement ces hypothèses, puisque les atteintes, dans chacun de ces domaines, ne correspondent pas forcément à un déficit des informations présumées prépondérantes (Caramazza et Shelton, 1998 ; Lambon Ralph, Howard, Nightingale et Ellis, 1998 ; Caramazza et Mahon, 2003 ). Cependant, des recherches chez l'adulte sain apportent, à l'aide de tâches et de procédures diverses, des arguments quant au poids relatif des types de propriétés selon le domaine. En outre, l'explication des dissociations observées a été plus récemment élargie en s'intéressant, d'une part, à la spécificité ou distinctivité des propriétés et, d'autre part, à leurs inter-corrélations. 
Evaluation du poids relatif des propriétés visuelles et fonctionnelles

Laws, Humber, Ramsey et McCarthy (1995), dans une tâche de vérification de propriétés, montrent que les adultes mettent plus de temps à vérifier les propositions portant sur les attributs visuels que fonctionnels/associatifs pour les objets fabriqués, mais ne trouvent pas de différence pour les animaux. Dans cette épreuve, selon Randall, Moss, Rodd, Greer, et Tyler (2004), la lecture du nom du concept activerait spontanément les divers attributs de ce concept, des plus centraux aux plus périphériques. Selon ce raisonnement, les attributs fonctionnels/associatifs étant vérifiés plus rapidement que les attributs visuels seraient plus caractéristiques des objets dans le domaine du non vivant. En revanche, dans le domaine du vivant, les attributs fonctionnels/associatifs seraient aussi centraux que les attributs visuels. Au contraire, Tyler et Moss (1997) montrent, à l'aide du paradigme d'amorçage sémantique automatique, que l'information fonctionnelle semble centrale dans les deux domaines. Dans une tâche de décision lexicale, les auteurs observent une supériorité de l'amorçage fonctionnel sur l'amorçage perceptif, équivalente pour les objets naturels et fabriqués.

Ces résultats contradictoires (voir aussi Sartori et Job, 1988 ; Farah et McClelland, 1991) peuvent s'expliquer par les différences de procédures mais aussi par les divergences, selon les auteurs, dans la définition des propriétés fonctionnelles. Les recherches de Garrard, Lambon Ralph, Hodges et Patterson (2001), McRae et Cree (2002), Cree et McRae (2003), dans lesquelles des adultes devaient produire les propriétés de divers concepts d'objets naturels et fabriqués, ont spécifiquement étudié cet aspect. La définition la plus restrictive des propriétés fonctionnelles n'inclut que les activités pour les animaux et l'utilisation pour les objets fabriqués. Elle exclut alors un grand nombre de propriétés fonctionnelles, en particulier les propriétés biologiques pour les objets naturels, ainsi que les liens contextuels et associatifs pour les deux types d'objets (Tyler et Moss, 1997). En génération de propriétés, selon la 
définition la plus restrictive, les propriétés perceptives/visuelles sont nettement plus nombreuses que les propriétés fonctionnelles parmi les objets naturels, mais en nombre à peu près équivalent parmi les objets fabriqués (Garrard et al., 2001 ; McRae et Cree, 2002). En revanche, plus la définition des propriétés fonctionnelles s'élargit allant jusqu'à inclure les liens contextuels et les connaissances encyclopédiques, plus les auteurs notent un abaissement de l'avantage, dans le cas des objets naturels, des propriétés perceptives/visuelles sur les propriétés fonctionnelles/associatives. Enfin, lorsque la définition inclut tout ce qui n'est pas perceptif, alors dans les deux domaines, les propriétés fonctionnelles sont plus nombreuses que les propriétés perceptives/visuelles (McRae et Cree, 2002).

Distinctivité des propriétés et inter-corrélations

Par ailleurs, l'organisation sémantique ne dépend pas seulement du nombre relatif des diverses propriétés des concepts mais de la quantité d'information qu'elles véhiculent. Les études déjà citées (Garrard et al., 2001; McRae et Cree, 2002; Cree et McRae, 2003) s'intéressent également à cet aspect relatif à la généralité ou spécificité des propriétés selon les domaines. Une propriété est dite générale quand elle est partagée par un grand nombre ou par l'ensemble des exemplaires d'une catégorie (par exemple, tous les véhicules servent à transporter). Au contraire, elle est dite spécifique, et donc distinctive, si elle est partagée par un nombre restreint d'objets d'une catégorie (peu d'animaux ont des rayures). Or, il apparaît que les attributs fonctionnels caractérisant le domaine du vivant, sont souvent généraux : tous les animaux et toutes les plantes respirent, se nourrissent, etc. En revanche, ceux qui caractérisent le domaine du non vivant sont souvent plus spécifiques : parmi les ustensiles de cuisine, peu d'objets coupent. Les normes recueillies chez l'adulte montrent effectivement que les propriétés distinctives sont nettement plus nombreuses pour les objets fabriqués que naturels (Garrard et al., 2001 ; McRae et Cree, 2002 ; Cree et McRae, 2003). Si l'on relie cet 
aspect avec la nature des propriétés, on peut alors envisager que les propriétés les plus distinctives soient surtout de type fonctionnel/associatif pour le non vivant et, au contraire, plus souvent de type perceptif/visuel pour le vivant.

Enfin, un troisième facteur relatif aux corrélations entre attributs est aussi susceptible d'influencer l'organisation des connaissances en mémoire (McRae, de Sa et Seidenberg, 1997 ; Randall et al., 2004). Les attributs des objets naturels seraient plus corrélés entre eux que ceux des objets fabriqués (McRae et al., 1997 ; Devlin, Gonnerman, Andersen et Seidenberg, 1998 ; Garrard et al., 2001 ; McRae et Cree, 2002). Pourtant, la différenciation entre domaines selon ce seul aspect n'est pas nette (Cree et McRae, 2003). Ce qui les différencierait, en revanche, serait la force des corrélations entre des propriétés perceptives/visuelles spécifiques et fonctionnelles/associatives de divers niveaux de généralité. Selon Tyler et Moss (1997), les propriétés perceptives générales des objets naturels sont fortement corrélées aux propriétés fonctionnelles biologiques (a des yeux et peut voir, a une bouche et se nourrit) alors que les propriétés perceptives distinctives (a une crinière) le sont beaucoup moins. A l'inverse, pour les objets fabriqués, c'est souvent la forme spécifique qui est associée à la fonction distinctive pour laquelle l'objet a été fabriqué (les outils qui coupent ont une lame). L'étude récente de Randall et al. (2004) chez des adultes sains montre effectivement que les propriétés générales sont plus fortement inter-corrélées dans le domaine du vivant que du non vivant tandis qu'à l'inverse, les propriétés spécifiques sont plus souvent et plus fortement inter-corrélées pour les objets fabriqués que naturels.

En résumé, les propriétés caractéristiques les plus corrélées à d'autres propriétés semblent majoritairement perceptives/visuelles et générales dans le cas des concepts d'objets naturels et plus souvent associatives/fonctionnelles et spécifiques dans le cas des concepts d'objets fabriqués chez l'adulte jeune. Aucune étude à notre connaissance ne fournit de données évaluant conjointement ces trois facteurs. Les données recueillies auprès de patients 
Alzheimer peuvent cependant apporter certains éléments de réponse pour comprendre le vieillissement normal.

Vieillissement de l'organisation conceptuelle

En effet, la dissociation vivant / non vivant a parfois été observée dans cette pathologie. Les travaux ont alors conduit les auteurs à proposer des modèles de détérioration de la mémoire sémantique (Devlin et al., 1998 ; Tyler, Moss, Durrant-Peatfield et Levy, 2000). Comme dans l'encéphalite herpétique, un profil fréquemment rapporté dans la démence de type Alzheimer (DTA) concerne une détérioration plus importante des concepts du vivant que du non vivant (Silveri, Daniele, Giustolisi et Gainotti, 1991; Fung, Chertkow, Murtha, Whatmough, Peloquin, Whitehead et Templeman, 2001; Zannino, Perri, Carlesimo, Pasqualetti et Caltagirone, 2002 ; Whatmough, Chertkow, Murtha, Templeman, Babins et Kelner, 2003), même si le profil inverse a déjà été observé (Capitani, Laiacona, Mahon et Caramazza, 2003). Si ce fait est maintenant reconnu, ses explications restent controversées. Comme nous l'avons énoncé, l'hypothèse classique de la SFT (Warrington et al., 1984) est aujourd'hui discutée au regard de facteurs complémentaires (distinctivité et inter-corrélation entre les propriétés) dont il faut tenir compte dans l'étude des troubles sémantiques (Cree et McRae, 2003). Par exemple, Zannino et al. (2002) ne trouvent pas de lien, chez des patients Alzheimer, entre le déficit spécifique relatif aux concepts d'objets naturels et une détérioration accrue de l'information perceptive ou fonctionnelle. Cependant, le niveau de spécificité des propriétés qui semble jouer un rôle modulateur majeur dans ces dissociations n'est pas pris en compte dans cette étude. A notre connaissance, une seule recherche a évalué l'implication conjointe du type et du niveau de distinctivité des propriétés en fonction du domaine dans la DTA (Roll-Carpentier, Bonthoux, Charnallet et Moreaud, 2003). Les résultats montrent globalement une atteinte du vivant expliquée par un déficit pour les propriétés visuelles et 
spécifiques de ces catégories avec une préservation des catégories d'objets fabriqués. Il est important de noter que l'atteinte d'un type de propriétés (visuelles ou fonctionnelles) n'apparaît qu'en lien avec le niveau de spécificité des propriétés. Ces données nous conduisent à formuler plusieurs hypothèses.

Tout d'abord, on peut s'attendre à un ralentissement global de l'accès aux propriétés des objets chez les personnes âgées par rapport aux adultes jeunes. On peut aussi de manière plus nuancée envisager des différences d'activation entre jeunes et âgés en fonction des facteurs précités. Les propriétés les plus caractéristiques, perceptives ou fonctionnelles, spécifiques ou générales, et les plus corrélées à d'autres resteraient plus accessibles lors du vieillissement et en cas d'atteinte cérébrale tandis que les propriétés les moins caractéristiques et les moins corrélées seraient plus sujettes à la détérioration.

Le but de cette étude est donc de déterminer quelles sont les propriétés des objets les plus sensibles au vieillissement normal dans chaque domaine. Nous pourrons alors déterminer si le vieillissement pathologique correspond à une forme accélérée du vieillissement normal ou à un profil particulier. Ces hypothèses sont testées ici à l'aide d'une épreuve de vérification de propriétés proposée à des adultes jeunes et âgés. Cette tâche très simple et utilisant un matériel très familier devrait conduire à des performances excellentes chez l'adulte sain (jeune ou âgé). Cependant, elle devrait engendrer des variations dans les temps de réponse selon l'âge, reflétant ainsi une plus ou moins grande facilité d'accès aux propriétés en fonction des variables étudiées, le domaine ainsi que le type et le niveau de spécificité des propriétés.

\section{Expérience}

La tâche de vérification de propriétés consiste à décider le plus rapidement possible si la propriété présentée est caractéristique ou pas du concept qui l'a précédée. Elle a été utilisée 
chez les patients atteints de désordres sémantiques (Moss, Tyler et Devlin, 2002 ; RollCarpentier et al. 2003) et aussi chez l'adulte sain (Laws et al., 1995 ; Randall et al., 2004). C'est la raison pour laquelle nous l'avons choisie pour étudier quelles propriétés sont plus ou moins facilement accessibles chez l'adulte âgé sain, comparé à l'adulte jeune. Comme exposé précédemment, on s'attend d'abord à un avantage global des propriétés fonctionnelles sur les propriétés visuelles, dans le cadre d'une définition large du terme fonctionnel. Cet avantage pour les propriétés fonctionnelles par rapport aux visuelles pourrait même être assez fort chez les personnes âgées si l'évolution est analogue dans le vieillissement normal et pathologique : en effet, on observe une détérioration massive des propriétés visuelles chez les patients Alzheimer sans trouble perceptif (Roll-Carpentier et al., 2003). De plus, nous avons vu que les attributs caractéristiques seraient surtout fonctionnels pour les objets fabriqués mais à la fois perceptifs et fonctionnels pour les objets naturels (McRae et Cree, 2002). Les participants devraient donc vérifier plus rapidement les propriétés fonctionnelles que visuelles des concepts d'objets fabriqués mais aussi rapidement les propriétés visuelles que fonctionnelles pour les concepts d'objets naturels, comme l'ont déjà montré Laws et ses collaborateurs (1995). Au vu des performances de patients Alzheimer (Roll-Carpentier et al., 2003), les propriétés visuelles du vivant pourraient devenir particulièrement difficiles d'accès chez les personnes âgées, sans doute parce qu'elles sont peu corrélées à d'autres caractéristiques.

Enfin, si l'on s'intéresse au niveau de spécificité des propriétés tel que l'ont proposé Tyler et Moss (1997, 2001), on s'attend à ce que les propriétés spécifiques des objets fabriqués soient plus faciles d'accès que celles des objets naturels, du fait de corrélations à la fois plus fréquentes et plus fortes ; c'est ce que trouvent effectivement Randall et ses collaborateurs (2004) chez les adultes jeunes. A l'inverse, pour la même raison, l'accès aux propriétés générales devrait être facilité pour les objets naturels plutôt que fabriqués. Sur ce dernier point, les résultats de ces auteurs (Randall et al., 2004) ne confirment pas la prédiction de 
Tyler et Moss puisque aucune différence entre domaines n'est observée pour les propriétés générales. Notre étude permettra de confirmer ou non les résultats de Randall et ses collaborateurs chez les adultes jeunes et de déterminer s'ils s'observent chez les adultes âgés. Pour notre part, comme énoncé précédemment, nous envisageons que les domaines se différencient si l'on tient compte à la fois du niveau (spécifique/général) et du type (visuel/fonctionnel) de propriété, ce qui n'a jamais été fait. Nous supposons en particulier que, si les propriétés les moins corrélées sont celles dont l'accessibilité diminue le plus avec l'avancée en âge, alors pour les propriétés visuelles du vivant, la vitesse d'accès chez les âgés, comparativement aux adultes jeunes, devrait être inférieure pour les propriétés spécifiques que générales. En effet, d'une part l'atteinte la plus fréquente dans le domaine du vivant, lors de la maladie d'Alzheimer comme dans les déficits spécifiques à une catégorie, concerne essentiellement l'identification des objets naturels et non leur appartenance à des catégories larges (animaux ou êtres vivants) et, d'autre part, les propriétés les plus fragiles chez les patients Alzheimer sont les propriétés spécifiques visuelles du vivant (Roll-Carpentier et al., 2003). De façon complémentaire, puisque les propriétés fonctionnelles, caractéristiques des concepts d'objets fabriqués, sont à la fois plus souvent spécifiques que générales et plus fortement corrélées à d'autres attributs dans ce domaine, cela permet d'attendre, en particulier chez les personnes âgées, un avantage pour les propriétés fonctionnelles spécifiques des objets fabriqués par rapport à celles des objets naturels.

Ces hypothèses sont testées ici à l'aide d'un questionnaire d'attributs dans lequel sont manipulés la nature des propriétés (fonctionnelles ou visuelles), leur niveau de spécificité (communes ou distinctives) et leur domaine d'appartenance (vivant ou non vivant) en fonction de l'âge des participants.

\section{Méthode}




\section{I.1. Participants}

Quinze adultes âgés et quinze adultes jeunes ont participé à l'expérience. Le groupe "âgés" se compose de 11 femmes et 4 hommes. La moyenne d'âge est de 78,5 ans (de 65 à 90 ans, écart-type : 8,7 ans). Ce sont des personnes autonomes vivant à leur domicile et ne présentant aucun signe de détérioration cognitive (MMS supérieur à 27). Elles ont été interrogées au sein d'espaces personnes âgées. Le groupe "jeunes" se compose de 9 hommes et 6 femmes. La moyenne d'âge est de 27,6 ans (de 19 à 35 ans, écart-type : 5,4 ans).

\section{I.2. Matériel}

Le questionnaire de propriétés est constitué de 192 affirmations composées d'un concept suivi d'une propriété qui peut être vraie ou fausse. Seize autres affirmations ont été utilisées lors d'une phase préalable de familiarisation. Pour la phase de test, il y a 24 concepts dont 12 objets naturels ( 3 fruits, 3 légumes et 6 animaux) et 12 objets fabriqués ( 3 meubles, 3 moyens de transport, 3 outils et 3 ustensiles de cuisine). La sélection dans chacune des catégories s'est faite sur la base de leur typicalité évaluée par d'autres auteurs (Rosch, 1975 ; Cordier, 1980 ; Hampton et Gardiner, 1983 ; Segalowitz et Poulin-Dubois, 1990). Pour éviter des difficultés d'accès liées au type d'objet, nous avons choisi les objets les plus typiques de chaque catégorie.

Les propriétés vraies sélectionnées sont des propriétés citées fréquemment par des adultes jeunes lors d'une tâche de génération de propriétés administrée précédemment (voir Annexe). Les propriétés fausses sont des propriétés vraies empruntées à un autre concept du même domaine pour la moitié des items et du domaine opposé pour l'autre moitié. Nous avons choisi une définition large des propriétés fonctionnelles puisqu'elles incluent l'utilisation des objets (ex : "sert à planter des clous"), les fonctions biologiques des entités vivantes (ex : "se reproduit"), les liens associatifs (ex: "se trouve dans la cuisine") et les connaissances 
encyclopédiques (ex: "pousse dans les pays chauds"). La longueur des propositions impliquant les propriétés (courtes ou longues) a été contre-balancée dans chaque condition. Les propriétés fonctionnelles débutent alternativement par "sert à", "pour" ou par un verbe et les propriétés perceptives par "a", "est" ou par un participe passé. Quatre types de propriétés ont été utilisés : visuelles ou fonctionnelles et spécifiques ou générales. Ainsi, chaque objet apparaît 8 fois. De ce fait, les 24 objets sont présentés aléatoirement au sein de 8 blocs dont l'ordre d'apparition est également randomisé.

\section{I.3. Procédure}

L'épreuve se déroule sur un ordinateur à l'aide du logiciel E-prime. Dans un premier temps, les participants lisent la consigne sur l'écran et l'expérimentateur apporte systématiquement un complément d'informations pour sa compréhension. La tâche est simple puisqu'elle requiert de décider le plus rapidement possible si la propriété présentée à l'écran est vraie ou pas pour le concept qui l'a précédée. La passation débute par 16 items de familiarisation qui donnent lieu à un feed-back. La phase test se déroule ensuite avec les 192 items sans feedback. Une pause dont la durée est gérée par le sujet se situe au milieu de l'expérience. Elle lui permet de détendre ses doigts et de relâcher son attention car les temps de défilement des items nécessitent une concentration importante. Le temps mis pour vérifier les phrases est un indicateur de la facilité d'accès aux propriétés correspondantes.

\section{Résultats}

L'objectif étant de tester l'accessibilité des différentes propriétés caractérisant les concepts, nous n'avons pris en compte que les temps de réponse correspondant aux propriétés vraies. Il est à noter cependant que le pourcentage de réponses correctes est très élevé aussi bien pour 
les propriétés vraies que fausses (respectivement $92.7 \%$ et $95.6 \%$ ) que pour les participants jeunes et âgés (respectivement $95.6 \%$ et $92.6 \%$ ).

Une analyse de variance a été conduite sur les temps de réponses avec l'âge (adultes jeunes et âgés) comme facteur inter sujets et le domaine (objets naturels et fabriqués), le type de propriétés (visuelles et fonctionnelles) et le niveau de spécificité des propriétés (propriétés générales et spécifiques) comme facteurs intra sujets. Les résultats apparaissent dans le tableau 1.

Tableau 1. Temps de réponse moyens (et écarts types) en fonction de l'âge, du domaine sémantique, de la nature et du niveau de spécificité des propriétés.

Mean response times (and standard deviations) as a function of age, semantic domain, type and specificity level of properties.

\begin{tabular}{cllllllll}
\hline & \multicolumn{3}{c}{ Vivant } & \multicolumn{3}{c}{ Non Vivant } \\
\hline & \multicolumn{2}{c}{ P. visuelles } & \multicolumn{2}{c}{ P. fonctionnelles } & \multicolumn{2}{c}{ P. visuelles } & \multicolumn{2}{c}{ P. fonctionnelles } \\
\hline & Gén. & Spéc. & Gén. & Spéc. & Gén. & Spéc. & Gén. & Spéc. \\
\hline \multirow{2}{*}{$\begin{array}{c}\text { Adultes } \\
\text { Jeunes }\end{array}$} & 1509 & 1709 & 1571 & 1769 & 1697 & 1690 & 1383 & 1523 \\
& $(397)$ & $(453)$ & $(386)$ & $(514)$ & $(528)$ & $(465)$ & $(310)$ & $(428)$ \\
\hline \multirow{2}{*}{$\begin{array}{c}\text { Adultes } \\
\text { Agés }\end{array}$} & 2133 & 2366 & 2094 & 2171 & 2219 & 2315 & 2004 & 1967 \\
& $(508)$ & $(567)$ & $(435)$ & $(540)$ & $(436)$ & $(505)$ & $(361)$ & $(337)$
\end{tabular}

Globalement, les adultes âgés répondent plus lentement (2159 ms) que les jeunes (1606 ms), $\mathrm{F}(1,28)=13,26, \mathrm{CMe}=1379426, \mathrm{p}<0,01$. Nous observons également un effet principal du domaine : les réponses sont globalement plus lentes pour les objets naturels (1915 ms) que fabriqués $(1850 \mathrm{~ms}), \mathrm{F}(1,28)=6,53, \mathrm{CME}=39363$, p<0,05 et du type de propriété: les participants mettent plus de temps pour vérifier les propriétés visuelles (1955 ms) que fonctionnelles $(1810 \mathrm{~ms})$ des différents concepts, $\mathrm{F}(1,28)=29,41, \mathrm{CMe}=42782, \mathrm{p}<0,001$. De plus, comme attendu, l'interaction entre les facteurs âge et type de propriétés est significative, $\mathrm{F}(1,28)=4,20, \mathrm{CMe}=42782, \mathrm{p}<0,05$ : la difficulté d'accès des propriétés visuelles par rapport 
aux propriétés fonctionnelles s'accentue avec l'âge. L'interaction entre le type de propriétés et le domaine est, elle aussi, significative, $F(1,28)=24,95, \mathrm{CMe}=32622, \mathrm{p}<0,001$. Comme le laissaient prévoir les données issues des tâches de génération de propriété, les participants vérifient plus rapidement les propriétés fonctionnelles $(1719 \mathrm{~ms})$ que visuelles $(1980 \mathrm{~ms})$ pour les objets fabriqués, $\mathrm{F}(1,28)=47,34, \mathrm{CMe}=43257$, $\mathrm{p}<0,001$, alors que cette différence n'est pas significative pour les objets naturels, $\mathrm{F}(1,28)<1, \mathrm{CMe}=32146$, (respectivement, $1901 \mathrm{~ms}$ et $1929 \mathrm{~ms})$.

De plus, la difficulté d'accès spécifique aux personnes âgées attendue pour les propriétés perceptives d'objets naturels semble confirmée puisque la différence de temps entre les propriétés fonctionnelles et visuelles pour ces objets est significative (respectivement, 2132 ms et $2249 \mathrm{~ms}$ ) dans ce groupe, $\mathrm{F}(1,28)=6,41, \mathrm{CMe}=32146, \mathrm{p}<0,05$, alors qu'elle ne l'est pas chez les adultes jeunes $\mathrm{F}(1,28)=1,71, \mathrm{CMe}=32146, \mathrm{p}=0.20$, (respectivement, $1670 \mathrm{~ms}$ et 1609 ms, voir Figure 1). Il faut noter que ce phénomène ne s'observe pas dans le cas des objets fabriqués. En effet, même si l'interaction impliquant l'âge, le domaine et le type de propriété n'est pas significative, $\mathrm{F}(1,28)=2,14, \mathrm{CMe}=32622$, $\mathrm{p}=0,15$, celle concernant $\mathrm{l}$ 'âge et le type de propriété l'est pour les objets naturels, $\mathrm{F}(1,28)=7,37, \mathrm{CMe}=32146, \mathrm{p}<0,05$, mais ne l'est pas pour les objets fabriqués, $\mathrm{F}(1,28)<1, \mathrm{CMe}=43257$.

\section{Insérer la Figure 1}

En ce qui concerne les hypothèses relatives au niveau de spécificité des propriétés, on observe un effet principal de ce facteur, $\mathrm{F}(1,28)=16,48, \mathrm{CMe}=45988, \mathrm{p}<0,001 \quad(1826 \mathrm{~ms}$ pour les propriétés générales et 1939 ms pour les propriétés spécifiques) et surtout une interaction entre ce facteur et le domaine conceptuel, $F(1,28)=11,96, \mathrm{CMe}=45988, \mathrm{p}<0,01$. En effet 
indépendamment du type de propriété, les adultes mettent globalement plus de temps pour vérifier les propriétés spécifiques du vivant (2004 ms) que celles du non vivant (1874 ms), $\mathrm{F}(1,28)=15,98, \mathrm{CMe}=31689, \mathrm{p}<0,001$, mais la différence entre les domaines n'est pas significative pour les propriétés générales (respectivement, 1827 ms et 1826 ms, $\mathrm{F}(1,28)<1$, $\mathrm{CMe}=28499$ ). Ces résultats s'observent aussi bien chez les adultes jeunes que âgés. En outre, l'hypothèse a priori concernant la plus grande fragilité avec le vieillissement des propriétés spécifiques visuelles plutôt que fonctionnelles dans le cas des objets naturels est confirmée, $\mathrm{F}(1,28)=6,51, \mathrm{CMe}=43751, \mathrm{p}<0,05$ (2366 ms pour les propriétés visuelles du vivant et 2170 ms pour les propriétés fonctionnelles), cette différence n'est pas significative chez les adultes jeunes, $\mathrm{F}(1,28)<1, \mathrm{CMe}=43751$ (respectivement, $1709 \mathrm{~ms}$ et $1769 \mathrm{~ms}$ ). Lorsque les mêmes comparaisons sont effectuées pour les objets fabriqués, l'accès plus lent aux propriétés spécifiques visuelles plutôt que fonctionnelles s'observe dans les deux groupes, $F(1,28)=6,87$, $\mathrm{CMe}=30641, \mathrm{p}<0,05$ pour les jeunes, $\mathrm{F}(1,28)=29,64, \mathrm{CMe}=30641, \mathrm{p}<0,001$ pour les âgés, ce qui est cohérent avec l'importance particulière des propriétés fonctionnelles spécifiques dans le domaine du non vivant. Effectivement, les propriétés fonctionnelles spécifiques, conformément à nos attentes, sont vérifiées plus rapidement pour les objets fabriqués que naturels, chez les jeunes, $\mathrm{F}(1,28)=14,13, \mathrm{CMe}=32173, \mathrm{p}<0,001$ (respectivement $1522 \mathrm{~ms}$ et 1769 ms), comme chez les âgés, $\mathrm{F}(1,28)=9,68, \mathrm{CMe}=32173$, p < 0,01 (respectivement 1967 ms et $2171 \mathrm{~ms}$ ). De façon complémentaire, les propriétés visuelles générales sont globalement d'accès plus facile pour les objets naturels que fabriqués, $\mathrm{F}(1,28)=7,81, \mathrm{CMe}=35905, \mathrm{p}<0,01$ et ceci, sans interaction entre le domaine, le type et le niveau de spécificité des propriétés, $\mathrm{F}(1,28)<1, \mathrm{CMe}=35616$. 
III. Discussion

La tâche de vérification de propriétés avait pour but de déterminer si la facilité d'accès aux propriétés, visuelles et fonctionnelles et, générales et spécifiques, évoluait de manière différenciée au cours du vieillissement, dans le domaine du vivant et dans celui du non vivant. Les résultats relatifs à l'avancée en âge permettront, dans un premier temps, de comparer les formes du vieillissement normal et pathologique. Ensuite, de façon plus prospective, ils seront discutés vis-à-vis de certaines hypothèses développementales relatives à la construction des concepts d'objets. Cependant, avant de discuter cette évolution, nous traiterons d'abord des effets se manifestant à la fois chez les adultes jeunes et âgés.

\section{III.1. Facilité d'accès aux différentes propriétés selon les domaines}

Globalement, le temps mis pour vérifier une propriété est plus court dans le cas des objets fabriqués que naturels, des propriétés fonctionnelles que visuelles, et générales que spécifiques. En outre, comme le laissaient prévoir les normes recueillies chez l'adulte sain (Garrard et al., 2001; McRae et Cree, 2002; Cree et McRae, 2003), les propriétés fonctionnelles sont vérifiées plus rapidement que les propriétés visuelles pour les objets fabriqués, alors que l'on n'observe pas de différence de temps pour les objets naturels. Ces résultats répliquent ceux obtenus par Laws et al. (1995) et les généralisent puisque dans l'étude conduite par ces auteurs, d'une part, seuls des adultes jeunes étaient évalués et, d'autre part, les objets naturels impliqués se limitaient aux animaux. Ainsi, les propriétés caractéristiques des concepts d'objets fabriqués, qui sont ici les plus facilement accessibles, semblent majoritairement de nature fonctionnelle. En revanche, les concepts d'objets naturels seraient caractérisés par les deux types de propriétés de façon équivalente.

Par ailleurs, indépendamment de leur type, la vérification est globalement plus rapide pour les propriétés spécifiques des objets fabriqués que naturels. Ces résultats confortent ceux de 
Randall et ses collaborateurs (2004) obtenu sur un échantillon de jeunes adultes et s'appliquent également ici aux adultes âgés. On peut cependant noter que ces auteurs prévoyaient aussi une différence entre les domaines pour les propriétés générales, ce qui n'est observé ni dans leur étude, ni dans la nôtre. En ce qui concerne les adultes jeunes, Randall et ses collaborateurs (2004) suggèrent que l'absence de lien entre la facilité d'accès aux propriétés générales et la fréquence et/ou la force des corrélations pourrait être due à la très grande rapidité de réponse pour ces propriétés : si les participants ont déjà atteint leur limite inférieure de temps de réponse pour les objets fabriqués, on n'observera pas de diminution supplémentaire du temps pour les objets naturels. Dans le même ordre d'idée, on peut noter que dans les épreuves de décision d'appartenance catégorielle, les catégories surordonnées des deux domaines sont généralement préservées chez les patients malgré des déficits au niveau de base, c'est-à-dire dans l'identification d'objets (Chertkow et Bub, 1990 ; Hodges, Salmon et Butters, 1992). Des recherches complémentaires sont en cours de réalisation pour déterminer si les propriétés générales ont réellement un statut particulier dans la structuration des connaissances catégorielles. Enfin, un résultat original est que les domaines se différencient si l'on tient compte à la fois du niveau (spécifique/général) et de la nature (visuelle/fonctionnelle) des propriétés. En effet, les participants accèdent plus rapidement aux propriétés à la fois fonctionnelles et spécifiques des objets fabriqués que naturels, ce qui appuie directement les propositions de Tyler et Moss (1997).

En résumé, en ce qui concerne l'organisation sémantique, les propriétés fonctionnelles seraient globalement plus accessibles, en particulier à un niveau spécifique et pour les catégories d'objets fabriqués alors que les propriétés perceptives, en revanche, semblent plus difficiles d'accès pour les deux domaines, en particulier à un niveau spécifique. 
III.2. Vitesse d'accès aux propriétés et vieillissement

On constate d'abord que la plus grande difficulté d'accès ${ }^{2}$ aux propriétés visuelles que fonctionnelles s'accentue avec l'âge, indépendamment des domaines. Ce type de résultat a également été observé dans une étude chez des patients Alzheimer, dont la discrimination perceptive avait été contrôlée (Roll-Carpentier et al., 2003). En outre, si sur l'ensemble des participants de notre étude, les propriétés perceptives des objets naturels ne sont pas vérifiées plus lentement que les propriétés fonctionnelles, ce résultat s'observe chez les personnes âgées. En ce qui concerne les données obtenues dans la démence de type Alzheimer, seules deux études de groupe ont étudié conjointement les effets du domaine et du type de propriété. Les résultats sont contradictoires puisque Zannino et ses collaborateurs (2002) ne trouvent pas de lien entre le déficit spécifique au vivant et le type de propriétés tandis que Roll-Carpentier et al. (2003) observent à la fois une atteinte du vivant et une difficulté particulière pour les propriétés visuelles spécifiques des objets de ce domaine. Or, l'étude de Zannino et al. (2002) ne tient pas compte du niveau de spécificité des propriétés, facteur qui semble pourtant un modulateur essentiel des dissociations. En outre, les auteurs ne précisent pas quelles propriétés fonctionnelles ils utilisent, ce qui pourrait aussi engendrer un biais puisque les résultats en termes d'atteinte des propriétés perceptives ou fonctionnelles divergent selon le type de définition choisi (Garrard et al., 2001 ; McRae et Cree, 2002 ; Cree et McRae, 2003). Quant aux études de cas portant sur les déficits spécifiques à une catégorie, Capitani et al. (2003) ont analysé 79 études de patients d'étiologies diverses présentant un déficit spécifique à une catégorie: pour 61 d'entre eux, celui-ci concerne le domaine du vivant. Sur les 16 études de cas ayant pris en compte le type de propriétés, 2 seulement rapportent une atteinte plus prononcée des propriétés perceptives que fonctionnelles/associatives et concerne des patients atteints d'encéphalite herpétique. Le seul patient Alzheimer de ce groupe (DB,

\footnotetext{
${ }^{2}$ Dans cette partie, les résultats sont rapportés en terme de difficulté d'accès de certaines propriétés par rapport à d'autres puisqu'ils renvoient à des effets du vieillissement. Au contraire, dans la partie précédente qui concerne l'organisation sémantique des adultes jeunes et âgés, ils ont été présentés en terme de facilitation relative.
} 
Lambon Ralph et al., 1998) présente, quant à lui, un déficit équivalent pour les propriétés perceptives et fonctionnelles/associatives.

Même si des données complémentaires semblent nécessaires pour pouvoir trancher sur cet aspect, il semble que l'organisation conceptuelle repose sur un ensemble de facteurs organisateurs, ce qui appuie l'hypothèse principale des modèles de la mémoire sémantique élaborés à partir de l'évaluation de patients atteints de DTA (Devlin et al., 1998 ; Tyler et al. (2000). En effet, selon ces modèles, les déficits conceptuels observés dans la démence de type Alzheimer sont le reflet d'une organisation des connaissances basée sur la distribution des propriétés au sein des différents domaines. Les études comportementales rapportent principalement une atteinte pour les catégories d'objets naturels dans cette pathologie (Gonnerman, Andersen, Devlin, Kempler et Seidenberg, 1997 ; Garrard, Lambon Ralph, Watson, Powis, Patterson et Hodges, 2001 ; Capitani et al., 2003).En outre, il semble que celle-ci s'associent chez ces patients à une altération des propriétés perceptives centrales dans ce domaine (Roll-Carpentier et al., 2003), ce que nous observons ici en terme de fragilité chez les personnes âgées.

Cette conclusion doit cependant être modulée en fonction du degré de spécificité des propriétés impliquées. En effet, la plus grande difficulté d'accès aux propriétés visuelles des objets naturels chez les adultes âgés, comparativement aux adultes jeunes, concerne particulièrement les propriétés distinctives. Ainsi, ces propriétés visuelles qui renvoient à des concepts d'objets naturels relativement spécifiques (des catégories de niveau de base) deviendraient difficiles d'accès avant les propriétés fonctionnelles. Cette même difficulté particulière a également été observée chez les patients Alzheimer (Roll-Carpentier et al., 2003), ce qui suggère une évolution analogue du vieillissement normal et pathologique. Complémentairement, les propriétés générales du vivant, visuelles et fonctionnelles, semblent résister de la même manière au vieillissement, qu'il soit normal ou pathologique. 
En résumé, cette expérience montre que les traits sémantiques qui deviennent plus difficiles d'accès lors du vieillissement normal sont également les premiers perturbés lors du vieillissement pathologique de type Alzheimer. Cela suggère une forme commune de vieillissement conceptuel, vieillissement accéléré dans le cas de la maladie d'Alzheimer. Ces résultats demandent bien sûr confirmation puisque, dans le cas du vieillissement normal, il n'existe pas de recherche à laquelle nous référer. Le seul résultat divergent entre les deux altérations concerne les propriétés fonctionnelles. Alors que leur accès à un niveau spécifique plutôt que général dans le cas des objets fabriqués est particulièrement difficile chez les patients Alzheimer (Roll-Carpentier et al., 2003), nous n’observons pas ce résultat chez les personnes âgées saines. Les propriétés fonctionnelles, spécifiques en particulier, apparaissent donc globalement plus résistantes au vieillissement normal et pathologique ce qui vient conforter, là encore, les propositions de Tyler et Moss (1997). L'hypothèse de ces auteurs, issue en fait d'arguments relatifs à la construction des concepts chez l'enfant, reflète l'apport actuel des modèles développementaux dans la compréhension du vieillissement de l'organisation conceptuelle.

\section{III.3. Catégorisation vie-entière : lien avec la construction des catégories}

En effet, une idée assez ancienne, issue des études psychométriques de l'intelligence et des recherches expérimentales sur les styles cognitifs, est qu'avec l'avancée en âge, on observe un processus de dédifférenciation associé à une plus grande rigidité de certains traitements cognitifs (Montangero, 1993). Ce phénomène est à l'origine de l'hypothèse de rétrogenèse selon laquelle les comportements ou les stratégies déclineraient dans l'ordre inverse de leur acquisition. Le fait qu'une information soit acquise tôt permet de penser qu'elle sera plus résistante au vieillissement. A l'inverse, les connaissances acquises plus tardivement seraient les premières touchées. Par exemple, chez des adultes sains âgés de 75 ans, l'âge 
d'acquisition des noms d'objets est la variable qui contribue le plus à l'explication des performances en dénomination d'images (Hogdson et Ellis, 1998): les premiers noms d'objets appris sont ceux qui permettent les meilleures performances (voir aussi Ukita, Abe et Yamada., 1999). De ce fait, les performances observées avec l'avancée en âge peuvent être confrontées aux résultats obtenus chez l'enfant et peuvent éventuellement suggérer de nouvelles hypothèses développementales.

Chez l'enfant, plusieurs propositions ont été avancées quant à la formation des concepts d'objets. Les bébés, dès l'âge de 3-4 mois, sont capables de différencier visuellement des chats et des chiens (Quinn, Eimas et Rosenkrantz, 1993), des chaises et des tables, des animaux et des meubles (Behl-Chadha, 1996). Selon Quinn et Eimas (1996, 2000), les premières représentations catégorielles d'objets seraient donc de nature perceptive. D'autres informations, issues en particulier des actions avec les objets et des interactions sociales viendraient ensuite enrichir ces représentations initiales, permettant graduellement la formation des concepts d'objets. A l'inverse, selon Mandler (1992, 2000), les représentations perceptives initiales ne peuvent conduire aux concepts. S'appuyant sur la théorie de Nelson (1985), cet auteur envisage que c'est grâce à la compréhension des actions dans lesquelles les objets sont impliqués, et donc à la construction de représentations généralisées d'événements ou schémas, que les bébés parviendraient précocement aux représentations conceptuelles d'objets. Pour notre part, nous défendons une hypothèse pluraliste (Bonthoux, Berger et Blaye, 2004 ; Bonthoux et al., 2003 ; Lautrey, 1990) selon laquelle les concepts se formeraient sur la base d'informations à la fois perceptives et relatives à l'action, ces deux types d'information pouvant être utilisées différemment selon les enfants et être plus ou moins efficaces selon les situations.

Les résultats chez les personnes âgées sont compatibles avec la proposition développementale de Mandler (1992, 2000). En effet, puisque les propriétés fonctionnelles, issues de la prise en 
compte des événements et des situations auxquels les objets prennent part, restent très accessibles au cours du vieillissement, on peut supposer qu'elles ont été prises en compte précocement et qu'elles sont primordiales pour les premières compréhensions conceptuelles. Les très jeunes enfants sont effectivement capables, dès l'âge de 14 mois, de généraliser certaines actions à d'autres objets d'une même catégorie (Mandler et McDonough, 1996, 1998), ce que les auteurs interprètent comme la prépondérance de la fonction des objets, mouvement et actions, par rapport à leur apparence, pour la formation des premiers concepts. Ces résultats développementaux, invoqués par Tyler et Moss (1997) pour appuyer leur hypothèse quant à la résistance des propriétés fonctionnelles chez les patients atteints de troubles sémantiques, permettent d'envisager des recherches complémentaires afin de mieux articuler développement et vieillissement conceptuel en lien avec les domaines sémantiques et les niveaux catégoriels. 
Bibliographie

Behl-Chadha G. - (1996) Basic-level and superordinate-like categorical representations in early infancy, $\underline{\text { Cognition, }} \underline{60}, 105-141$.

Bonthoux F., Berger C., Blaye A. - (2004) Naissance et développement des concepts chez l'enfant. Catégoriser pour comprendre, Paris, Dunod.

Bonthoux F., Scheuner N., Roll N. - (2003) Des modes de construction des catégories différenciés selon le domaine. Eléments de réponse chez l'enfant et l'adulte âgé, Cognition, Brain, Behavior, $\underline{7}, 91-109$.

Capitani E., Laiacona M., Mahon B., Caramazza A. - (2003) What are the facts of semantic category-specific deficits? A critical review of the clinical evidence, $\underline{\text { Cognitive }}$ neuropsychology, 20 (3/4/5/6), 213-261.

Caramazza A., Mahon B.Z. - (2003) The organization of conceptual knowledge: The evidence from category-specific semantic deficits, Trends in Cognitive Sciences, $\underline{7}$, 354-361.

Caramazza A., Shelton J.R. - (1998) Domain-specific knowledge systems in the brain : The animate-inanimate distinction, Journal of Cognitive Neuroscience, 10, 1-35.

Chertkow H., Bub D. - (1990) Semantic memory loss in dementia of Alzheimer's type. What do various measures measure? Brain, $\underline{113,397-417 .}$

Cordier F. - (1980) Gradients de prototypie pour cinq catégories sémantiques, $\underline{\text { Psychologie }}$ Française, 25, 211-219.

Cree G.S., McRae, K. - (2003) Analyzing the factors underlying the structure and computation of the meaning of chipmunk, cherry, chisel, cheese, and cello (and many other such concrete nouns), Journal of Experimental Psychology: General, 132, 163-201

Devlin J.T., Gonnerman L.M., Anderson E.S., Seidenberg M.S. - (1998) Category-specific semantic deficits in focal and widespread brain damage: A computational account, Journal of Cognitive Neuroscience, $\underline{10}, 77-94$. 
Farah M.J., McClelland J.L. - (1991) A computational model of semantic memory impairment : Modality specificity and emergent category specificity, $\underline{\text { Journal of Experimental }}$ Psychology: General, 120, 339-357.

Fung T.D., Chertkow H., Murtha S., Whatmough C., Peloquin L., Whitehead V., Templeman F. - (2001) The spectrum of category effects in object and action knowledge in dementia of the Alzheimer type, Neuropsychology, 15 (3), 371-379.

Garrard P., Lambon Ralph M.A., Hodges J.R., Patterson K. - (2001) Prototypicality, distinctiveness and intercorrelation: Analyses of the semantic attributes of living and

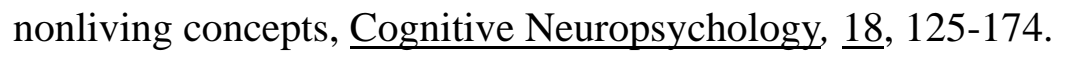

Garrard P., Lambon Ralph M.A., Watson P.C., Powis J., Patterson K., Hodges J.R. - (2001) Longitudinal profiles of semantic impairment for living and nonliving concepts in DTA,

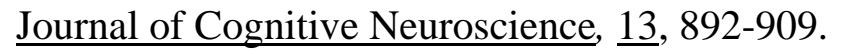

Gonnerman L.M., Andersen E.S., Devlin J.T., Kempler D., Seidenberg M.S. - (1997) Double dissociation of semantic categories in Alzheimer's disease, Brain and Language, $\underline{57}, 254-279$. Hampton J.A., Gardiner M.M. - (1983) Measures of internal category structure: A correlational analysis of normative data, British Journal of Psychology, $\underline{74}$, 491-516.

Hodges J.R., Salmon D.P., Butters N. - (1992) Semantic memory impairment in Alzheimer's disease: Failure of access or degraded knowledge? Neuropsychologia, 30, 301-314.

Hodgson C., Ellis A.W. - (1998) Last in, first to go: Age of acquisition and naming in the elderly, Brain and Language, $\underline{64}$, 146-163.

Lambon Ralph M.A., Howard D., Nightingale G., Ellis A.W. - (1998) Are living and nonliving category-specific deficits causally linked to impaired perceptual or associative knowledge? Evidence from a category-specific double dissociation, Neurocase, 4, 311-338.

Lautrey J. - (1990) Des conceptions unitaires aux conceptions pluralistes du développement

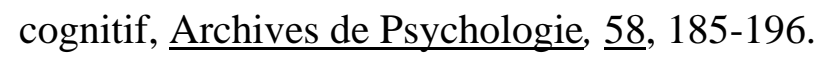


Laws K.R., Humber S.A., Ramsey D.J.C., McCarthy R.A. - (1995) Probing sensory and associative semantics for animals and objects in normal subjects, Memory, $\underline{3}$, 397-408.

Mandler J. M. - (1992) How to build a baby: Conceptual primitives, Psychological Review, 99, 587-604.

Mandler J.M. - (2000) Perceptual and conceptual processes in infancy, Journal of Cognition and Development, 1, 3-36.

Mandler J.M., McDonough L. - (1996) Drinking and driving don't mix : Inductive generalization in infancy, Cognition, 59, 307-335.

Mandler J.M., McDonough L. - (1998) Studies in inductive inference in infancy, Cognitive Psychology, 37, 60-96.

Montangero J. - (1993) Vieillissement non pathologique, in J. Montangero (Edit.), Psychologie de la personne âgée. Aspects neuropsychologiques, cognitifs et cliniques de vieillissement, Paris, PUF.

McRae K., Cree G.S. - (2002) Factors underlying category-specific semantic deficits, in E.M.E. Forde et G. Humphreys (Edit.), Category-specificity in mind and brain, East Sussex (UK), Psychology Press.

McRae K., De Sa V.R., Seidenberg M.S. - (1997) On the nature and scope of featural representations of word meaning, Journal of Experimental Psychology: General, 126, 99-130. Moss H.E.., Tyler L.K., Devlin, J. - (2002) The emergence of category-specific deficits in a distributed semantic system, in E.M.E. Forde et G. Humphreys (Edit.), Category-specificity in brain and mind, East Sussex (UK), Psychology Press, 115-148.

Nelson K. - (1985) Making sense. Development of meaning in early childhood, New York, Academic Press. 
Quinn P.C., Eimas P.D. - (1996) Perceptual organization and categorization in young infants, in C. Rovee-Collier et L.P. Lipsitt (Edit.), Advances in infancy research, $\mathrm{t}$ X, Norwood (NJ), $1-36$.

Quinn P. C., Eimas P. D. - (2000) The emergence of category representations during infancy: Are separate perceptual and conceptual processes required? Journal of Cognition and Development, $\underline{1}$, 55-61.

Quinn P.C., Eimas P.D., Rosenkrantz S.L. - (1993) Evidence for representations of perceptually similar natural categories by 3-month-old and 4-month-old infants, Perception, $\underline{22}, 463-475$.

Randall B., Moss H.E., Rodd J.M., Greer M., Tyler L.K. - (2004) Distinctiveness and correlation in conceptual structure: Behavioral and computational studies, $\underline{\text { Journal of }}$ Experimental Psychology: Learning, Memory, and Cognition, 30, 393-406.

Roll-Carpentier N., Bonthoux F., Charnallet A., Moreaud O. - (2003) L'apport des modèles développementaux dans la compréhension des troubles sémantiques de la maladie d'Alzheimer, Revue Neurologique, 159, 5S41.

Rosch E. - (1975) Cognitive representation of semantic categories. Journal of Experimental Psychology: General, 104, 192-233.

Sartori G., Job R. - (1988) The oysters with four legs : a neuropsychological study on the interaction between vision and semantic information, Cognitive Neuropsychology, $\underline{5}, 105-$ 132.

Silveri M.C., Daniele A., Giustolisi L. et Gainotti G. - (1991) Dissociation between knowledge of living and non living things in dementia of the Alzheimer type, Neurology, $\underline{41,} 545-546$. 
Segalowitz N., Poulin-Dubois D. - (1990) The structure of categories: typicality gradients, perceived linguistic familiarity and cross-linguistic comparison. Cahiers de Psychologie Cognitive/European Bulletin of Cognitive Psychology, 10, 491-512.

Tyler L.K., Moss H.E. - (1997) Functional properties of concepts : Studies of normal and brain-damage patients, Cognitive Neuropsychology, 14, 511-545.

Tyler L.K., Moss H.E. - (2001) Toward a distributed account of conceptual knowledge. Trends in Cognitive Sciences, $\underline{5}, 244-252$.

Tyler L.K., Moss H.E., Durrand-Pietfield M.R., Levy J.P. - (2000) Conceptual structure of concepts : A distributed account of categoy-specific deficits, Brain and Langage, 75, 195-231. Ukita H., Abe K., Yamada J. - (1999) Late acquired words in childhood are lost zarlier in primary progressive aphasia, Brain and Language, $\underline{70}$, 205-219.

Warrington E.K., McCarthy R.A. - (1983) Category specific access dysphasia. Brain, 106, 859-878.

Warrington E.K., McCarthy R.A. - (1987) Categories of knowledge : Further fractionations and an attempted integration, $\underline{\text { Brain, }} \underline{110}, 1273-1296$.

Warrington E.K., Shallice T. - (1984) Category specific semantic impairments, Brain, 107, 829-853.

Whatmough C., Chertkow H., Murtha S., Templeman D., Babins L. et Kelner N. - (2003) The semantic category effects increases with worsening anomia in Alzheimer's type dementia, Brain and language, $\underline{84}, 134-147$.

Zannino G.D., Perri R., Carlesimo G.A., Pasqualetti P. et Caltagirone C. - (2002) Categoryspecific impairment in patients with Alzheimer's disease as a function of disease severity : A cross-sectional investigation, Neuropsychologia, $\underline{40}$, 2268-2279. 


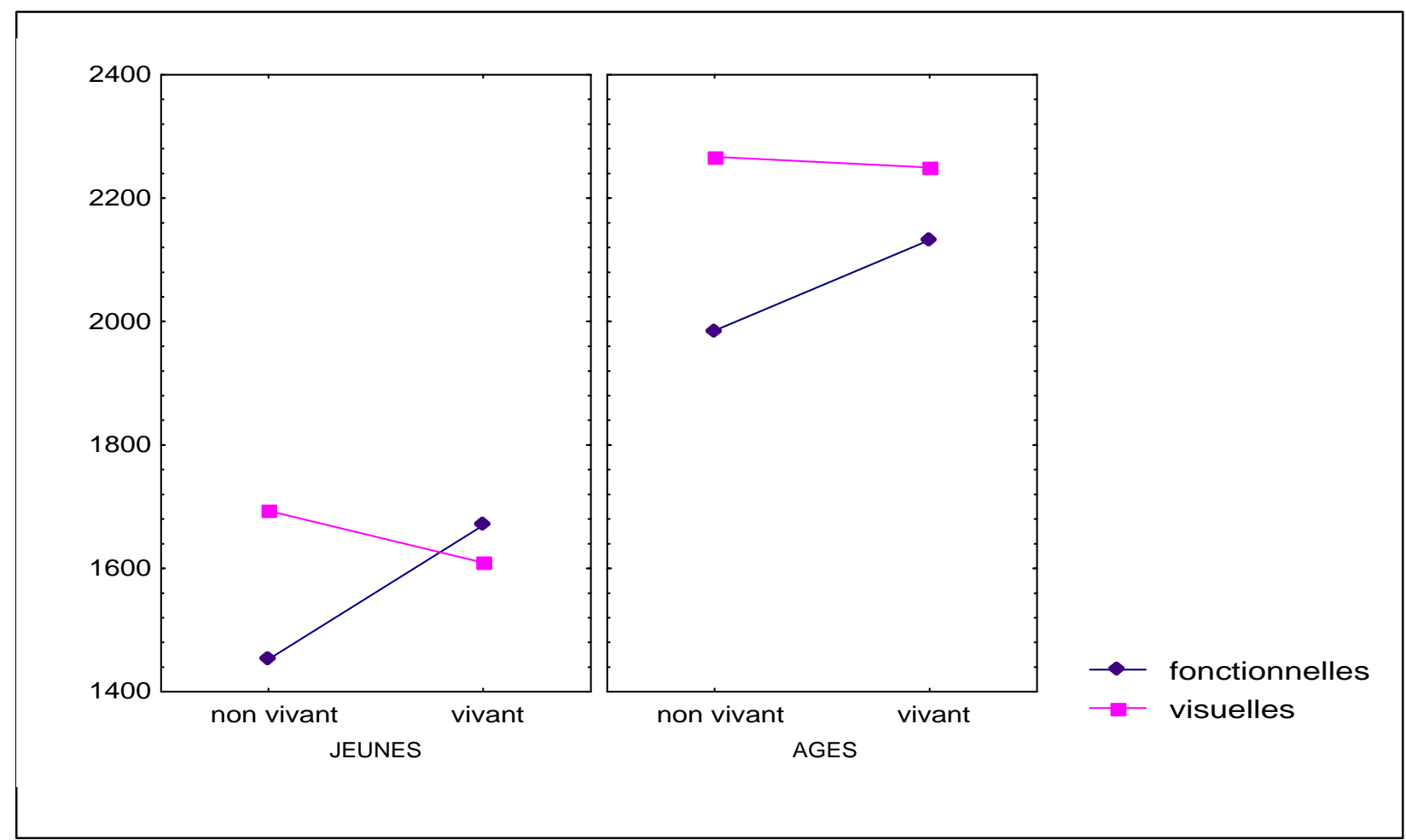

Fig. 1 : Temps de réponse moyen (ms) en fonction de l'âge, du domaine sémantique et du type de propriété

Mean response times ( $m s)$ as a function of age, semantic domain, and property type 


\section{Titres des figures}

Fig. 1: Temps de réponse moyen (ms) en fonction de l'âge, du domaine sémantique et du type de propriété

Mean response times (ms) as a function of age, semantic domain, and property type 
Annexe

Liste des propriétés fonctionnelles

\begin{tabular}{|c|c|c|}
\hline & générales & spécifiques \\
\hline avion & transporte des gens & se déplace dans l'air \\
\hline buffet & sert à ranger & se trouve dans la cuisine \\
\hline canapé & pour s'asseoir & pour se relaxer \\
\hline casserole & pour cuisiner & pour réchauffer \\
\hline couteau & sert à table & sert à couper \\
\hline marteau & sert à réparer & plante des clous \\
\hline poêle & pour cuire les aliments & sert à faire frire \\
\hline scie & est dangereuse & pour couper le bois \\
\hline table & pour travailler & pour poser des choses \\
\hline tournevis & pour bricoler & sert à visser \\
\hline train & permet de voyager & roule sur des rails \\
\hline voiture & sert à se déplacer & fonctionne à l'essence \\
\hline aigle & se nourrit d'animaux & vit en altitude \\
\hline banane & s'épluche & pousse dans les pays chauds \\
\hline carotte & pousse & se mange rapée \\
\hline chat & se reproduit & peut griffer \\
\hline cheval & peut sauter & peut être monté \\
\hline chien & peut courir & est fidèle \\
\hline fourmi & vit en groupe & peut piquer \\
\hline mouche & peut voler & transmet des microbes \\
\hline orange & utile à l'organisme & s'utilise en jus \\
\hline poire & se mange en dessert & sert à faire des tartes \\
\hline pomme de terre & se cuisine & se mange en frites \\
\hline tomate & se mange en entrée & pour faire des sauces \\
\hline
\end{tabular}


Liste des propriétés visuelles

\begin{tabular}{|c|c|c|}
\hline Concept & générales & spécifiques \\
\hline avion & est grand & a des ailes \\
\hline buffet & a des portes & a un tiroir \\
\hline canapé & a un dossier & est garni de coussins \\
\hline casserole & de forme cylindrique & est profonde \\
\hline couteau & est pointu & a une lame \\
\hline marteau & est fait de deux parties & a une tête carrée \\
\hline poêle & est plate & a des bords minces \\
\hline scie & de forme allongée & a des dents \\
\hline table & souvent rectangulaire & a un plateau \\
\hline tournevis & a un manche & formé d'une tige métallique \\
\hline train & a des fenêtres & formé de wagons \\
\hline voiture & a des sièges & a un volant \\
\hline aigle & a des yeux & a des serres \\
\hline banane & de forme courbée & a une couleur jaune \\
\hline carotte & est longue & est orange \\
\hline chat & a des poils & a des moustaches \\
\hline cheval & a des oreilles & a des sabots \\
\hline chien & a une queue & a une gueule \\
\hline fourmi & a des pattes & a des antennes \\
\hline mouche & est petite & a des gros yeux \\
\hline orange & est ronde & a une peau granuleuse \\
\hline poire & est jaune ou verte & a une peau mince \\
\hline pomme de terre & a une peau & est beige \\
\hline tomate & est rouge & a une peau lisse \\
\hline
\end{tabular}

